Check for updates

Cite this: RSC Adv., 2019, 9, 4344

\title{
Improved dielectric stability of epoxy composites with ultralow boron nitride loading $\dagger$
}

\begin{abstract}
Xiuwu Fu, Yiping Guo, (D)* Qi Du, Lin Guan and Sibo He
Polymer-based composites have attracted increasing interest and been widely used in the field of electronic devices, but they are limited due to their low working temperature and dielectric instability. To date, there are few reports on improving the dielectric stability of polymer-based composites. Herein, threedimensional boron nitride/nylon 66 (3D BN/PA66) aerogels are facilely prepared for the first time by freeze-drying without templates. The BN/PA66 composites are prepared by infiltrating 3D BN/PA66 aerogels with the epoxy. The thermal conductivity of the 3D BN/PA66 composite increases to $0.6 \mathrm{~W} \mathrm{~m}^{-1}$ $\mathrm{K}^{-1}$ due to the formation of a BN microplatelet thermal conduction network at an ultralow BN loading of 4 vol\%, which is about 5-fold and 3-fold higher than that of neat epoxy and random BN/EP composite with the same BN loading. Meanwhile, the dielectric constant variation of the BN/PA66 composites is only $6 \%$, showing much better stability in the dielectric properties than neat epoxy and random BN/EP composites over the temperature range $25-200{ }^{\circ} \mathrm{C}$. Our research provides a new strategy to prepare the 3D BN aerogels and proves that the 3D BN/PA66 aerogel is a potential platform for preparing polymer-based composites with excellent dielectric stability.
\end{abstract}

Received 12th December 2018 Accepted 28th January 2019

DOI: 10.1039/c8ra10211b

rsc.li/rsc-advances usually used to improve the compatibility between fillers and polymer matrix. ${ }^{\mathbf{1 8 - 2 0}}$ However, the effect of surface modification and functionalization on thermal properties and dielectric stability of polymer-based composites are undesired at high temperature.

Recently, a lot of novel strategies have been developed to improve the thermal conductivity or dielectric stability of the polymer-based composites. Shao et al. ${ }^{21}$ have prepared a hybrid three-dimensional boron nitride nanosheet (BNNS)/graphene framework as thermal conductive filler in the polymeric composites. They observed a synergistic effect between BNNSs and graphene, which can significantly improve the thermal conductivity of neat nylon 6. Dai et al. ${ }^{22}$ have fabricated a rigid three-dimensional structure composed of silicon carbide nanowire and graphene sheets by using the high frequency heating process. The thermal conductivity of the composites is significantly raised, approximately a 10-fold enhancement in comparison with that of neat polyimide. Although the threedimensional graphene structure can significantly improve the thermal conductivity of the composites, it cannot effectively suppress dielectric loss of the composites due to its high electric conductivity. The dielectric loss of the composites containing conductive phases (e.g. graphene, metal particles) is too high to meet the requirements of electronic device. ${ }^{23-25}$ Threedimensional boron nitride (3D $\mathrm{BN})$ aerogels give consideration to both high-efficiency thermal conductivity and excellent electrical insulating property, which are more suitable for preparing the polymer-based composites with excellent dielectric stability. Zeng et al. ${ }^{26}$ have successfully prepared the 3D BN
State Key Laboratory of Metal Matrix Composites, School of Materials Science and Engineering, Shanghai Jiao Tong University, Shanghai 200240, People's Republic of China. E-mail: ypguo@sjtu.edu.cn; Fax: +86-21-34202749; Tel: +86-21-54738972

$\dagger$ Electronic supplementary information (ESI) available. See DOI: 10.1039/c8ra10211b 
aerogel by introducing the PVA. However, the preparation process of the $3 \mathrm{D} \mathrm{BN}$ network composites is complicated and requires pressing with a mould, which may be difficult to prepare large or special size polymer-based composites. $\mathrm{Hu}$ et $a{ }^{27}{ }^{27}$ have successfully prepared the $3 \mathrm{D}$ BN aerogel by introducing the sodium methylcellulose. However, the introduction of the sodium ion will increase the dielectric loss of the polymer-based composites. Li et al. ${ }^{5}$ have fabricated crosslinked polymer nanocomposites containing BN nanosheets, which exhibits stable dielectric constant and low dielectric loss over a wide temperature and frequency range. Although fillers introduced for the preparation of 3D BN aerogels may lead to an increase in the dielectric loss of the polymer-based composites, ${ }^{27}$ the $3 \mathrm{D}$ BN aerogels prepared by reasonable strategy could be ideal functional fillers to prepare the polymer-based composites with excellent dielectric stability due to their excellent thermal and electrical insulating properties. ${ }^{5}$

The nylon 66 (PA66) is a thermoplastic resin material and one of the most widely used engineering materials, owing to their excellent mechanical and thermal properties, good electrical insulation properties and easy processing. ${ }^{28,29}$ We have found that PA66 pellets can be dissolved in formic acid to form an aerogel after the freeze-drying without templates, which can be used as a three-dimensional framework. The functional fillers can adhere to the PA66 aerogel to form a thermal conduction network at an ultralow loading. However, the 3D PA66 aerogels prepared by the freeze-drying have not been reported yet. Although the thermal conductivity of epoxy resin (model no. E-51) is low (around $0.15 \mathrm{~W} \mathrm{~m}^{-1} \mathrm{~K}^{-1}$ ), epoxy E-51 is widely used as the matrix for the polymer-based composites in the field of electronic devices due to their good electrical insulation, easy casting and excellent mechanical strength. ${ }^{30-33}$ To date, there are few reports on improving the dielectric stability of epoxy-based composites. The epoxy-based composites can possess much higher thermal conductivity by introducing a large amount of high thermal conductivity fillers, while excessive fillers will hinder the crosslinking and polymerization of the epoxy and form lots of interface with the epoxy matrix, which not only increases processing difficulty but also increases the dielectric loss of the epoxy composites..$^{34,35}$

In this study, the 3D BN/PA66 aerogels have been prepared by the freeze-drying without templates, and $\mathrm{BN}$ microplatelets can adhere to 3D PA66 aerogels to form a continuous thermal conduction network at an ultralow loading. The BN/PA66 composites are prepared by infiltrating 3D BN/PA66 aerogels with epoxy resin. Moreover, the dielectric and thermal properties of the 3D BN/PA66 composites are investigated.

\section{Experimental}

\subsection{Materials}

The PA66 pellets were obtained from Tengfei Co., Ltd with average size of 2-4 mm. Epoxy resin (model no. E-51) was purchased from Dow Chemical Co. Acetone and formic acid were purchased from Aladdin Industries Ltd and used as solvent. The 4,4-diaminodiphenylmethane (DDM) was purchased from Sinopharm Chemical Reagent Co., Ltd and used as the curing agent. Hexagonal BN microplatelets were supplied by Yaan Bestry Performance Materials Co., Ltd and used as received.

\subsection{Preparation of $3 \mathrm{D}$ BN/PA66 aerogels}

$2.0 \mathrm{~g}$ of PA66 pellets were dissolved into $20 \mathrm{~mL}$ of formic acid by stirring at $60{ }^{\circ} \mathrm{C}$. The BN/PA66 slurries were prepared by dispersing different volume fraction of $\mathrm{BN}$ microplatelets into the formic acid solution of PA66. The slurries were processed by ball-milling for $24 \mathrm{~h}$ at $400 \mathrm{rpm}$. Subsequently, the slurries were poured into a mould. Through the freeze-drying (Freeze-dryer, FD-1C-50, China) at minus $100{ }^{\circ} \mathrm{C}$ and $20 \mathrm{~Pa}$ for $48 \mathrm{~h}$, the $3 \mathrm{D}$ BN/PA66 aerogels were successfully prepared.

\subsection{Preparation of 3D BN/PA66 composites}

Epoxy E-51 is used as the matrix due to its excellent electrical insulation, mechanical property and chemical resistance, which is very suitable for application in the electronic devices. DDM was dissolved into acetone by stirring at $70{ }^{\circ} \mathrm{C}$. A certain amount of liquid epoxy resin was mixed with DDM by stirring for $30 \mathrm{~min}$ at room temperature (epoxy E-51/DDM $=5: 1$, wt/wt). The mixture was poured into the 3D BN/PA66 aerogels to prepare the 3D BN/PA66 composites, and then the composites were transferred into vacuum oven to remove the bubbles at $10^{4} \mathrm{~Pa}$ and $70{ }^{\circ} \mathrm{C}$. The epoxy resin can be effectively infused into the 3D BN/ PA66 aerogels due to capillary force and low pressure atmosphere. Finally, the 3D BN/PA66 composites were cured at $80{ }^{\circ} \mathrm{C}$ for $2 \mathrm{~h}$ and $120^{\circ} \mathrm{C}$ for $4 \mathrm{~h}$. According to the volume ratio of the $\mathrm{BN}$, the as-prepared three-dimensional composites are named as 3D BN/PA66- $X-Y$ composites, where $X$ stands for the volume fraction of the $\mathrm{BN}$ in the aerogel and $Y$ stands for the volume fraction of the $\mathrm{BN}$ in the whole composite. The conversion of volume fraction and mass fraction of $\mathrm{BN}$ microplatelets in the whole composite are shown in the Table S1.† For comparison, epoxy resin directly mixed with 4 vol\% of BN microplatelets without 3D PA66 aerogels was also prepared and denoted as random BN/EP-4 composites. The schematic fabrication process of the 3D BN/PA66 composite is shown in Fig. 1.

\subsection{Characterizations}

The morphologies of hexagonal BN microplatelets, 3D PA66 aerogels and cross section of the 3D BN/PA66 composites were observed by scanning electron microscope (S-4800, HITACHI, Japan). The morphology of hexagonal BN microplatelets was also characterized by transmission electron microscope (JEM2100F, JEOL, Japan). The phase structure of BN microplatelets was characterized by X-ray diffraction (XRD, D/MAX2550/PC, Rigaku Co.) using $\mathrm{Cu} \mathrm{K \alpha}$ radiation from $15^{\circ}$ to $80^{\circ}$ at scanning rate of $5^{\circ} \mathrm{min}^{-1}$ under $35 \mathrm{kV}$ and $200 \mathrm{~mA}$. The thermal diffusivity $\left(D, \mathrm{~mm}^{2} \mathrm{~s}^{-1}\right)$ of the 3D BN/PA66 composites was measured by the laser flash technique (LFA 457). The thermal conductivity $\left(\lambda, \mathrm{W} \mathrm{m}^{-1} \mathrm{~K}^{-1}\right)$ of the 3D BN/PA66 composites was calculated through the following formula:

$$
\lambda=D \rho C_{\mathrm{p}}
$$


where $\rho$ stands for the density of 3D BN/PA66 composites, which is calculated by water displacement method, and $C_{\mathrm{p}}$ stands for the specific heat of 3D BN/PA66 composites. The samples were prepared into disc-shaped forms with a diameter of $12.5 \pm$ $0.2 \mathrm{~mm}$ and a thickness of $1.0 \mathrm{~mm}$. Before testing, the surface of the sample was treated by graphite spraying to ensure uniform temperature on the front and back of the sample. The degradation temperature $\left(T_{\mathrm{d}}\right)$ of $3 \mathrm{D} \mathrm{BN} / \mathrm{PA} 66$ composites was measured by Pyris1 TGA from $25{ }^{\circ} \mathrm{C}$ to $800{ }^{\circ} \mathrm{C}$ at heating rate of $10{ }^{\circ} \mathrm{C} \min ^{-1}$ under nitrogen atmosphere. The glass transition temperature $\left(T_{\mathrm{g}}\right)$ of 3D BN/PA66 composites was measured by Perkin-Elmer DMA 8000 from $0{ }^{\circ} \mathrm{C}$ to $200{ }^{\circ} \mathrm{C}$ at heating rate of $3{ }^{\circ} \mathrm{C} \min ^{-1}$ and $1 \mathrm{~Hz}$. Dielectric constant and dielectric loss of 3D BN/PA66 composites were characterized by the impedance analyzer (Agilent 4990A) from $10^{2} \mathrm{~Hz}$ to $10^{6} \mathrm{~Hz}$ at room temperature and from $25{ }^{\circ} \mathrm{C}$ to $200{ }^{\circ} \mathrm{C}$ at $10^{4} \mathrm{~Hz}$, respectively. Before measurement, silver paste was applied on both sizes of the composite films to ensure their good electric conduction.

\section{Results and discussion}

The XRD patterns of BN microplatelets are shown in Fig. 2, and the diffraction peaks of any impurities are not observed within the range of $15-80^{\circ}$. The result of the XRD indicates that the $\mathrm{BN}$ microplatelet is of high purity.

The microscopic surface morphology of the BN microplatelet was characterized by SEM and TEM as shown in Fig. 3(a) and (b). The average size of BN microplatelets ranges from 3 to $5 \mu \mathrm{m}$, and the thickness is in the range of 100-300 $\mathrm{nm}$. The 3D PA66 aerogel is macroscopically composed of slice stacks with a porous structure, as shown in Fig. S1. $\dagger$ From the Fig. 3(c) and (d), the 3D PA66 aerogels possess good mechanical strength and compactness. In addition, the external shape of 3D PA66

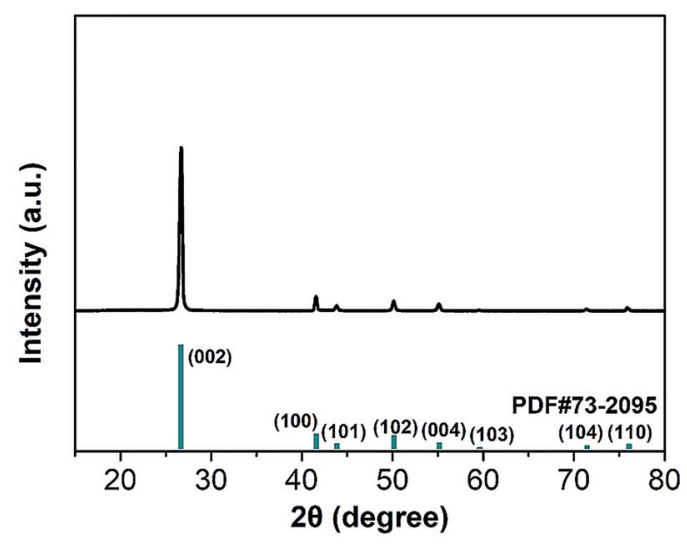

Fig. 2 The XRD spectra of the BN microplatelets.

aerogels can be adjusted according to the preparation process to meet different requirements for the shape and size of the electronic device. As shown in Fig. 3(e), the 3D PA66 aerogel also possesses an abundance of internal interface. The BN microplatelets can easily adhere to the PA66 aerogel to form a continuous thermal conduction network at an ultralow loading. Moreover, the dispersion of BN microplatelets in PA66 aerogel was characterized by SEM as shown in Fig. S2. $\dagger$ As illustrated in Fig. 3(f), the fracture section of the 3D BN/PA66-00 composite is relatively smooth, showing the brittleness characteristics of the epoxy resin. Meanwhile, it can be observed that 3D PA66 aerogels can be well coated by the epoxy resin, which is attributed to the good compatibility between 3D PA66 aerogels and epoxy resin. The well-compatibility between epoxy resin and 3D PA66 aerogels plays a crucial role in improving dielectric and thermal properties of 3D BN/PA66 composites.

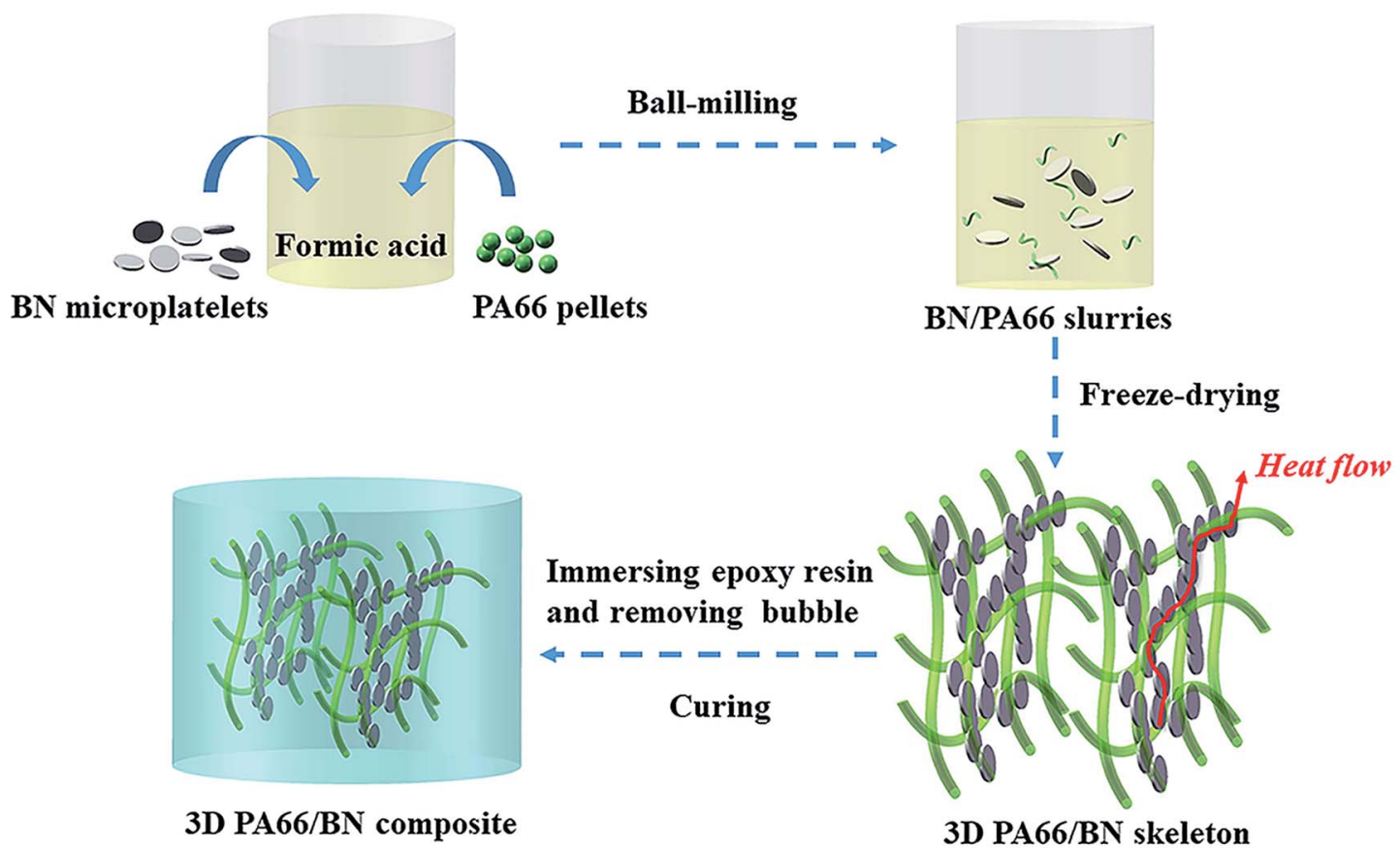

Fig. 1 The schematic illustration of preparation process for the 3D BN/PA66 composites. 

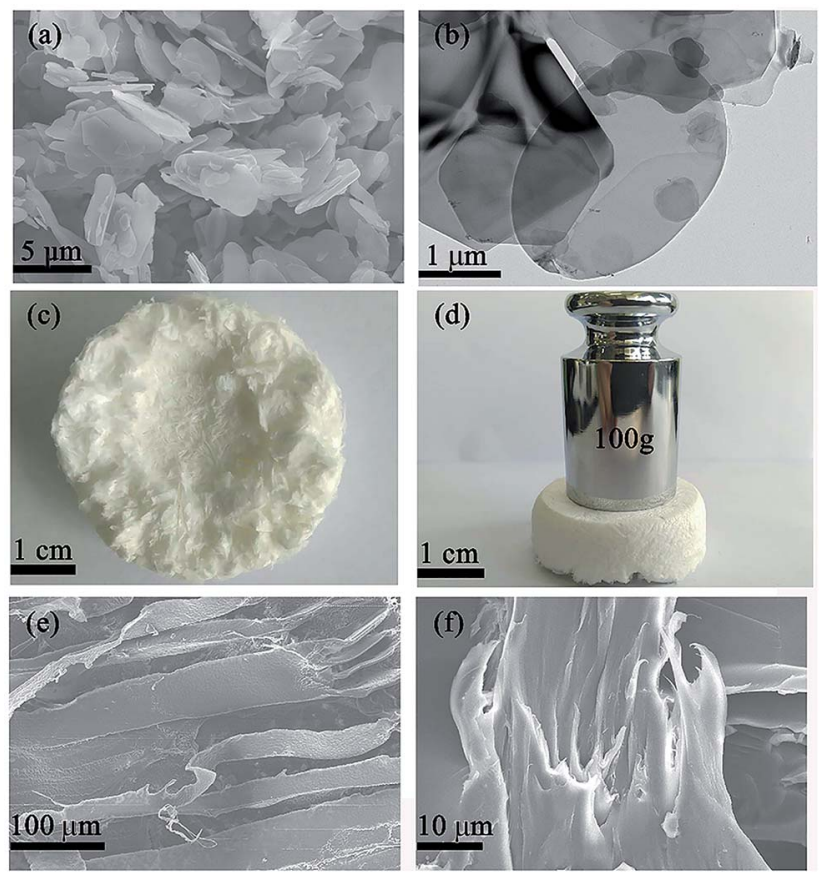

Fig. 3 (a) SEM image of the BN microplatelets; (b) TEM image of the BN microplatelets; (c) and (d) the optical image of pristine 3D PA66 aerogel; (e) SEM image of pristine 3D PA66 aerogel; (f) cross section SEM image of pristine 3D BN/PA66-0-0 composite.

The degradation temperature $\left(T_{\mathrm{d}}\right)$ is one of the most important parameters to characterize the thermal stability of polymer-based composites. The $T_{\mathrm{d}}$ of 3D BN/PA66 composites were measured in nitrogen atmosphere as illustrated in Fig. 4. In the temperature range of $330-450{ }^{\circ} \mathrm{C}$, the mass of $3 \mathrm{D} \mathrm{BN} /$ PA66 composites drops sharply due to the decomposition of the epoxy matrix in this temperature range ${ }^{36}$ From the Table 1 , the $T_{5}$ of all 3D BN/PA66 composites are above $330^{\circ} \mathrm{C}$, which is higher than that of the neat epoxy. The effect of BN/PA66 aerogels on the $T_{\mathrm{d}}$ can be ascribed to the following aspects: on one hand, the PA66 aerogel can improve the thermal stability of the composite due to their excellent thermal stability. Besides, PA66 aerogel and epoxy matrix can form a partially crosslinked

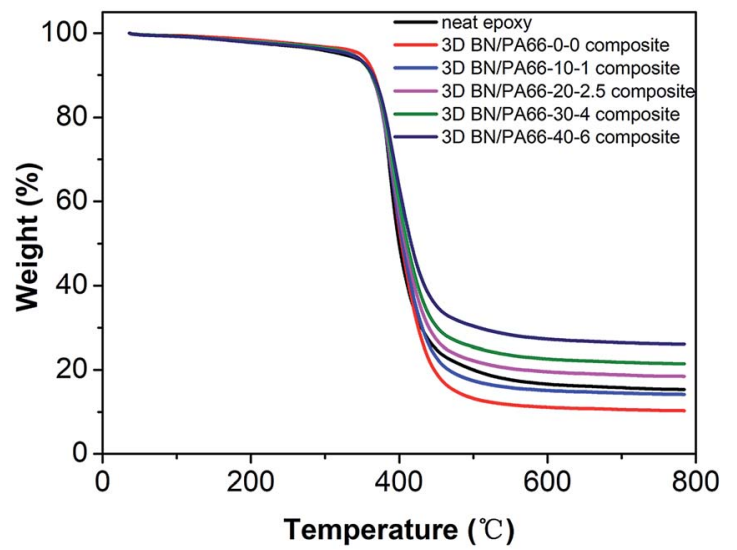

Fig. 4 The TGA curve of the neat epoxy and 3D BN/PA66 composites in the $\mathrm{N}_{2}$ atmosphere.
Table 1 Degradation temperature $\left(T_{\mathrm{d}}\right)$ and glass transition temperature $\left(T_{\mathrm{g}}\right)$ of the neat epoxy and 3D BN/PA66 composites $^{a}$

\begin{tabular}{lll}
\hline Sample & $T_{5} /{ }^{\circ} \mathrm{C}$ & $T_{\mathrm{g}} /{ }^{\circ} \mathrm{C}$ \\
\hline Neat epoxy & 320 & 115 \\
3D BN/PA66-0-0 composite & 347 & 127 \\
3D BN/PA66-10-1 composite & 336 & 125 \\
3D BN/PA66-20-2.5 composite & 335 & 124 \\
3D BN/PA66-30-4 composite & 334 & 123 \\
3D BN/PA66-40-6 composite & 332 & 120
\end{tabular}

${ }^{a} T_{5}$ indicates the temperature at which the mass loss of the materials is $5 \%$.

structure, further reducing the degradation rate. On the other hand, the increase in BN content makes adverse effect on the compatibility between PA66 aerogel and epoxy matrix, which will reduce the $T_{\mathrm{d}}$ of the BN/PA66 composites. The excellent thermal stability suggests the 3D BN/PA66 aerogel is a potential candidate for preparing high-temperature polymer-based composites.

The glass transition temperature $\left(T_{\mathrm{g}}\right)$ in this work is defined as the temperature at the peak of the loss factor curves, as shown in Fig. 5 and Table 1 . The $T_{\mathrm{g}}$ of all 3D BN/PA66 composites are above $120{ }^{\circ} \mathrm{C}$, which is higher than that of the neat epoxy. The 3D PA66 aerogels constrain the segmental mobility of polymer chain and thus lead to an increase in the $T_{\mathrm{g}}$ of 3D BN/PA66 composites. However, as the increase of the BN content, the $T_{\mathrm{g}}$ value of $3 \mathrm{D}$ BN/PA66 composites slightly reduces. The $\mathrm{BN}$ microplatelets introduce the additional free volume inside epoxy matrix, which improves the segmental mobility of polymer chain and thus leads to reduction in $T_{\mathrm{g}}$ value. ${ }^{27}$ Meanwhile, the only one loss factor peak of 3D BN/PA66 composites is observed from $0{ }^{\circ} \mathrm{C}$ to $200{ }^{\circ} \mathrm{C}$, which indicates the good compatibility between 3D BN/PA66 aerogel and epoxy matrix.

The thermal conductivity of the 3D BN/PA66 composite was investigated over the temperature range $25-200^{\circ} \mathrm{C}$ as illustrated in Fig. 6. The thermal conductivity of the neat epoxy and random BN/EP-4 composite are about $0.15 \mathrm{~W} \mathrm{~m}^{-1} \mathrm{~K}^{-1}$ and

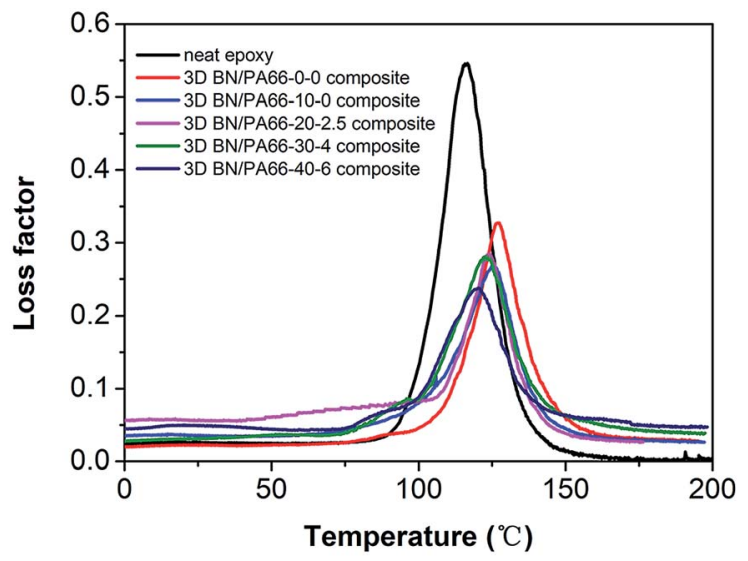

Fig. 5 The loss factor as a function of temperature of the neat epoxy and 3D BN/PA66 composites. 
$0.2 \mathrm{~W} \mathrm{~m}^{-1} \mathrm{~K}^{-1}$, respectively. The $\mathrm{BN}$ microplatelets are far from enough to form the continuous thermal conduction network in random BN/EP-4 composite at an ultralow loading of 4 vol\%. However, a large amount of randomly distributed BN microplatelets will not only cause serious agglomeration but also prevent epoxy resin from curing and cross-linking, which makes adverse effect on the dielectric stability of the composites. From the Fig. 6, the thermal conductivity of 3D BN/PA66-20-2.5 composite is about $0.3 \mathrm{~W} \mathrm{~m}^{-1} \mathrm{~K}^{-1}$, which is slightly higher than that of neat epoxy and random BN/EP-4 composite. As shown in Fig. 7(a) and (b), since the BN content is too low, a continuous thermal conduction network can't be formed throughout the composite. However, it can be observed the thermal conductivity of 3D BN/PA66-30-4 composite significantly increases up to $0.6 \mathrm{~W} \mathrm{~m} \mathrm{~m}^{-1} \mathrm{~K}^{-1}$, which is about 5-fold and 3-fold higher than that of neat epoxy and random BN/EP-4 composite. As shown in Fig. 7(c) and (d), the continuous thermal conduction networks have been effectively formed along the 3D PA66 aerogel in the whole composites at an ultralow BN loading of 4 vol\%. According to the percolation theory, when the content of $\mathrm{BN}$ microplatelets exceeds the percolation threshold, the increase in BN content will no longer have such significant effect on the thermal conductivity of the 3D BN/PA66 composite. Therefore, the thermal conductivity of 3D BN/PA66-40-6 composite slightly increases compared with that of 3D BN/PA66-30-4 composite. It is also well-noted that the thermal conductivity of 3D BN/PA66 composites basically remains stable over the temperature range $25-200{ }^{\circ} \mathrm{C}$ (Table $\left.\mathrm{S} 2 \dagger\right){ }^{30}$ Table 2 summarizes the thermal conductivity of the previously reported epoxy-based composites (model no. E-51) using different kinds of high thermal conductivity materials as fillers. From the Table 2, the thermal conductivity of low density polyethylene (LDPE)/BN/epoxy composite is $0.65 \mathrm{~W} \mathrm{~m}^{-1} \mathrm{~K}^{-1}$, which is similar to that of $3 \mathrm{D}$ BN/PA66-30-4 composite. As shown in Fig. 3(f), it can be observed that the compatibility between epoxy and 3D PA66 aerogel is excellent. There are no clear interfaces and microcracks between 3D PA66 aerogel and epoxy. However, the compatibility between the LDPE and epoxy is poor. There are clear interfaces and microcracks between LDPE and epoxy,

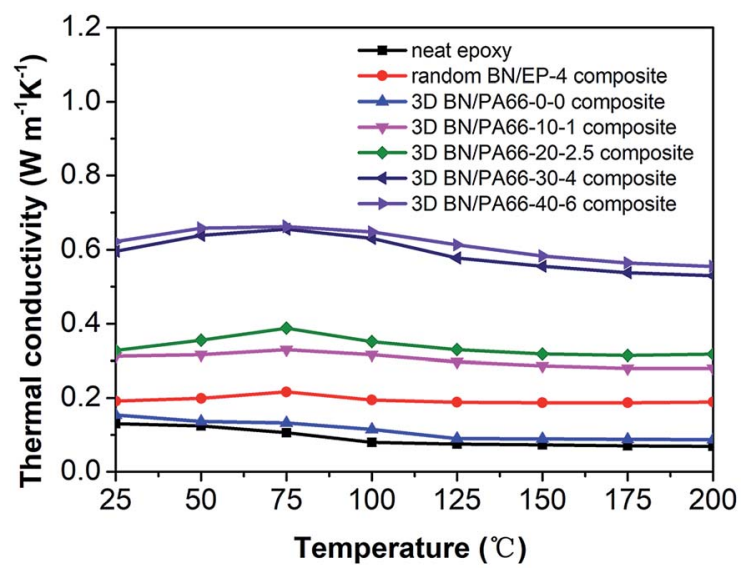

Fig. 6 The thermal conductivity as a function of temperature of the neat epoxy, random BN/PA66-4 composite and 3D BN/PA66 composites.

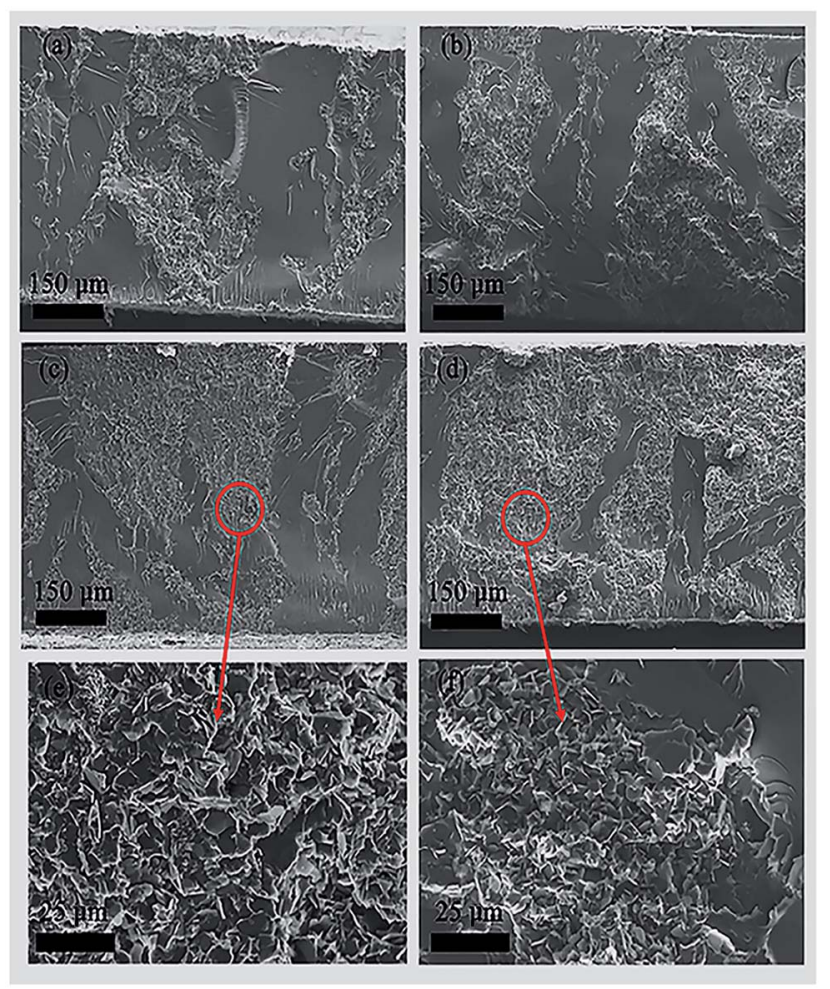

Fig. 7 The cross section SEM images of the (a) 3D BN/PA66-10-1 composites; (b) 3D BN/PA66-20-2.5 composites; (c) and (e) 3D BN/ PA66-30-4 composites; (d) and (f) 3D BN/PA66-40-6 composites.

which could make negative impact on the mechanical property and dielectric stability of the composites. ${ }^{37}$

The frequency dependence of dielectric constant of 3D BN/ PA66 composites with different volume fractions of BN microplatelets are shown in Fig. 8(a). The dielectric constant of the neat epoxy and random BN/EP-4 composite are about 3.55 and 4.32 over the frequency range $10^{2}$ to $10^{6} \mathrm{~Hz}$ at room temperature (RT, $25{ }^{\circ} \mathrm{C}$ ), respectively. The dielectric constant of 3D BN/PA66 composites remains stable in the frequency range of $10^{2}$ to $10^{6} \mathrm{~Hz}$, the specific values are shown in Table S3. $\dagger$ In addition, the dielectric constant of 3D BN/PA66 composites slightly improves as the loading of $\mathrm{BN}$ microplatelets increases. As shown in Fig. 8(b), the dielectric loss of the neat epoxy and random BN/EP-4 composite are about 0.023 and 0.035 over the frequency range $10^{2}$ to $10^{6} \mathrm{~Hz}$ at RT, respectively. Moreover, the dielectric loss of 3D BN/PA66 composites also remains stable in the frequency range of $10^{2}$ to $10^{6} \mathrm{~Hz}$, and the specific values are shown in Table. S3. $\dagger$ The weak frequency dependence of dielectric properties is mainly attributed to the wellcompatibility between 3D BN/PA66 aerogel and epoxy matrix, which can effectively suppresses the dielectric loss of the $\mathrm{BN} /$ PA66 composite.

The temperature dependence of dielectric properties of 3D BN/PA66 composites are shown in Fig. 9, and the specific values are shown in Table S4. $\dagger$ As shown in Fig. 9(a), the dielectric constant of the neat epoxy, random BN/EP-4 composite and 3D BN/PA66-20-2.5 composites significantly varies over the temperature range $25-200{ }^{\circ} \mathrm{C}$ at $10^{4} \mathrm{~Hz}$. From the Fig. 9(c), the 
Table 2 Comparison of thermal conductivity of this work with the previously reported epoxy E51-based composites

\begin{tabular}{|c|c|c|c|}
\hline Sample & $\begin{array}{l}\text { Filler loading } \\
\text { in the whole composite }\end{array}$ & $\mathrm{TC}\left(\mathrm{W} \mathrm{m}^{-1} \mathrm{~K}^{-1}\right)$ & Ref. \\
\hline Graphene/epoxy-E-51 & $0.5 \mathrm{wt} \%$ & 0.25 & 30 \\
\hline BN/LDPE/epoxy-E51 ${ }^{b}$ & 50 vol $\%$ & 0.65 & 37 \\
\hline AIN/LDPE/epoxy-E51 ${ }^{b}$ & $50 \mathrm{vol} \%$ & 0.68 & 37 \\
\hline PES/GNPs/epoxy-E51 ${ }^{c}$ & 15 vol $\%$ & 0.85 & 38 \\
\hline
\end{tabular}

${ }^{a}$ The MWCNT is multi-walled carbon nanotubes. ${ }^{b}$ The LDPE is low density polyethylene. ${ }^{c}$ The PES is polyethersulphone; the GNPs is graphite nanoplatelets.
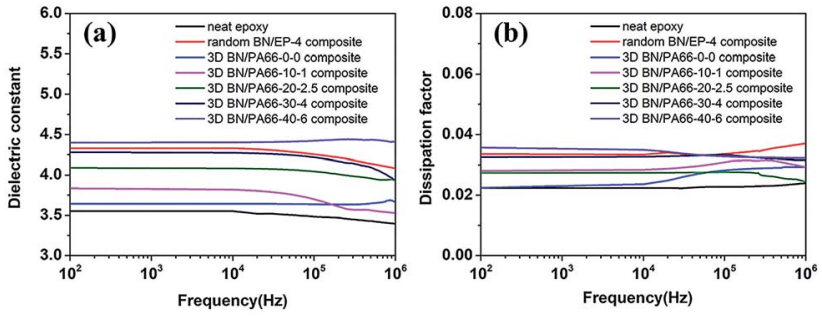

Fig. 8 Frequency dependence of dielectric constant and dielectric loss ( $a$ and $b$ ) of the neat epoxy, random BN/EP-4 composite and 3D BN/PA66 composites at room temperature.

variation of dielectric constant of the neat epoxy, random $\mathrm{BN} /$ EP-4 composite and 3D BN/PA66-20-2.5 composite are 48\%, $38 \%$ and $37 \%$, respectively. The temperature dependence of dielectric loss of 3D BN/PA66 composites are shown in Fig. 9(b). It can be observed the dielectric loss of neat epoxy, random $\mathrm{BN} /$ EP-4 composite and 3D BN/PA66-20-2.5 composite also severely varies with temperature. From the Fig. 9(d), the dielectric loss of neat epoxy, random BN/EP-4 composite and 3D BN/PA66-20-2.5 composites are $0.025,0.031$ and 0.029 at RT, which increases up
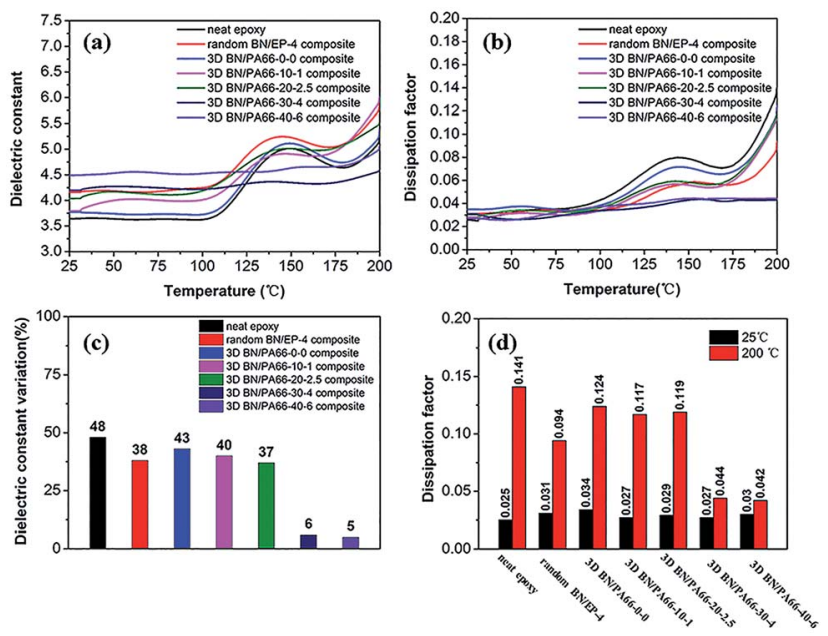

Fig. 9 Temperature dependence of dielectric constant and dielectric loss ( $a$ and $b$ ) of the neat epoxy, random BN/EP-4 composite and 3D BN/PA66 composites at $10^{4} \mathrm{~Hz}$, (c) the variation of dielectric constant over the temperature range $25-200{ }^{\circ} \mathrm{C}$, (d) the specific value of dielectric loss over the temperature range $25-200{ }^{\circ} \mathrm{C}$ at $10^{4} \mathrm{~Hz}$. to $0.141,0.094$ and 0.119 at $200{ }^{\circ} \mathrm{C}$, respectively. Since no continuous thermal conduction network has been formed throughout the composite, heat accumulates inside the composite and cannot be effectively conducted to the outside. Therefore, the dielectric constant and dielectric loss of these composites significantly vary with temperature, especially when temperature approaches the glass transition temperature of the composite. In the previous works, a large amount of BN microplatelets are directly added into epoxy matrix to improve its thermal conductivity. However, a lot of BN microplatelets will also hinder the crosslinking and polymerization of the epoxy and create plenty of interfaces with epoxy matrix, which leads to an increase in the dielectric loss of the epoxy-based composites. $^{34,35}$ As shown in the Fig. 9(c), the 3D BN/PA66-304 and BN/PA66-40-6 composites show the excellent stability of dielectric constant. The variation of dielectric constant of 3D $\mathrm{BN} / \mathrm{PA} 66-30-4$ composites is only $6 \%$, which is much lower than $38 \%$ of random $\mathrm{BN} / \mathrm{EP}-4$ composite. Meanwhile, 3D BN/PA6630-4 composite shows weak temperature dependence of dielectric loss in comparison with the random BN/EP-4 composite. From the Fig. 9(d), the dielectric loss of 3D BN/ PA66-30-4 composites are 0.027 at RT, which remains 0.044 at $200{ }^{\circ} \mathrm{C}$. The improved dielectric stability of the composites is mainly attributed to the following aspects: on the one hand, BN microplatelets can form a continuous thermal conduction network along the PA66 aerogel throughout the composite at an ultralow loading. The heat can be effectively conducted, and does not accumulate inside the composites. On the other hand, an ultralow loading of $\mathrm{BN}$ microplatelets can guarantee wellcompatibility between BN/PA66 aerogel and epoxy matrix, thus suppressing the dielectric loss.

It should be pointed out that in addition to the epoxy resin, 3D BN/PA66 aerogel also has good compatibility with a variety of polymers, such as polyurethane, polymethyl methacrylate and silicon rubber, etc. According to the different demands of application, the composites can be obtained by infiltrating $3 \mathrm{D}$ BN/PA66 aerogel with the different kinds of polymer matrices.

\section{Conclusions}

The high mechanical strength 3D BN/PA66 aerogel has been facilely prepared by the freeze-drying without templates, and BN microplatelets can uniformly adhere to 3D PA66 aerogel to form 
a continuous thermal conductivity network at an ultralow loading of $4 \mathrm{vol} \%$. The 3D BN/PA66 composites are obtained by infiltrating 3D BN/PA66 aerogel with epoxy resin (model no. E51). The degradation temperature and glass transition temperature of 3D BN/PA66 composites are above $330{ }^{\circ} \mathrm{C}$ and $120{ }^{\circ} \mathrm{C}$, respectively. For 3D BN/PA66-30-4 composites, the thermal conductivity is $0.6 \mathrm{~W} \mathrm{~m}{ }^{-1} \mathrm{~K}^{-1}$, which is about 5-fold and 3-fold higher than that of the neat epoxy and random BN/EP-4 composite. Meanwhile, 3D BN/PA66-30-4 composite shows the weak frequency and temperature dependence of dielectric properties. The dielectric constant and dielectric loss of 3D BN/ PA66-30-4 composite are about 4.28 and 0.032 over the frequency range $10^{2}$ to $10^{6} \mathrm{~Hz}$ at RT. In addition, due to the continuous thermal conduction network formed by BN microplatelets along 3D PA66 aerogels throughout the composite, the variation of dielectric constant of the 3D BN/PA66-30-4 composite is only $6 \%$, which is much lower than $38 \%$ of random $\mathrm{BN} / \mathrm{EP}-4$ composite, and the dielectric loss is below 0.044 over temperature range $25-200{ }^{\circ} \mathrm{C}$ at $10^{4} \mathrm{~Hz}$. The results indicate that 3D BN/PA66 aerogel is a potential platform for preparing the polymer-based composites with excellent dielectric stability.

\section{Conflicts of interest}

There are no conflicts to declare.

\section{Acknowledgements}

This work was supported by the Natural Science Foundation of China (No. 51332009 and 11474199). The Instrumental Analysis Centre of Shanghai Jiao Tong University is sincerely acknowledged for assisting with the relevant analyses.

\section{Notes and references}

1 M. A. Alam, M. H. Azarian and M. G. Pecht, J. Electron. Mater., 2013, 42, 1101-1107.

2 H. Zhao, L. Zhang, M.-H. Yang, Z.-M. Dang and J. Bai, Appl. Phys. Lett., 2015, 106, 092904.

3 W. Xu, Y. Ding, S. Jiang, L. Chen, X. Liao and H. Hou, Mater. Lett., 2014, 135, 158-161.

4 Y.-H. Zhang, Z.-M. Dang, J. H. Xin, W. A. Daoud, J.-H. Ji, Y. Liu, B. Fei, Y. Li, J. Wu, S. Yang and L.-F. Li, Macromol. Rapid Commun., 2005, 26, 1473-1477.

5 Q. Li, L. Chen, M. R. Gadinski, S. Zhang, G. Zhang, H. U. Li, E. Iagodkine, A. Haque, L.-Q. Chen, T. N. Jackson and Q. Wang, Nature, 2015, 523, 576.

6 F. Qi, N. Chen and Q. Wang, Mater. Des., 2018, 143, 72-80.

7 S.-Q. Wu, J.-W. Wang, J. Shao, L. Wei, R.-K. Ge and H. Ren, Mater. Des., 2018, 146, 208-218.

8 L. Fang, C. Wu, R. Qian, L. Xie, K. Yang and P. Jiang, RSC Adv., 2014, 4, 21010-21017.

9 K. Yang and M. Gu, Composites, Part A, 2010, 41, 215-221. 10 M. Du, B. Guo and D. Jia, Eur. Polym. J., 2006, 42, 1362-1369. 11 M. J. Biercuk, M. C. Llaguno, M. Radosavljevic, J. K. Hyun, A. T. Johnson and J. E. Fischer, Appl. Phys. Lett., 2002, 80, 2767-2769.
12 F. H. Gojny, M. H. G. Wichmann, B. Fiedler, I. A. Kinloch, W. Bauhofer, A. H. Windle and K. Schulte, Polymer, 2006, 47, 2036-2045.

13 X. Zhang, C. L. Pint, M. H. Lee, B. E. Schubert, A. Jamshidi, K. Takei, H. Ko, A. Gillies, R. Bardhan, J. J. Urban, M. Wu, R. Fearing and A. Javey, Nano Lett., 2011, 11, 3239-3244.

14 S. Wang, Y. Cheng, R. Wang, J. Sun and L. Gao, ACS Appl. Mater. Interfaces, 2014, 6, 6481-6486.

15 C.-C. Teng, C.-C. M. Ma, C.-H. Lu, S.-Y. Yang, S.-H. Lee, M.-C. Hsiao, M.-Y. Yen, K.-C. Chiou and T.-M. Lee, Carbon, 2011, 49, 5107-5116.

16 A. A. Balandin, Nat. Mater., 2011, 10, 569.

17 S. Vadukumpully, J. Paul, N. Mahanta and S. Valiyaveettil, Carbon, 2011, 49, 198-205.

18 H. Tang, Z. Ma, J. Zhong, J. Yang, R. Zhao and X. Liu, Colloids Surf., A, 2011, 384, 311-317.

19 C. Yang, Y. Lin and C. W. Nan, Carbon, 2009, 47, 1096-1101. 20 A. Xie, Y. Wang, P. Jiang, S. Li and X. Huang, Compos. Sci. Technol., 2018, 154, 154-164.

21 L. Shao, L. Shi, X. Li, N. Song and P. Ding, Compos. Sci. Technol., 2016, 135, 83-91.

22 W. Dai, J. Yu, Y. Wang, Y. Song, F. E. Alam, K. Nishimura, C.-T. Lin and N. Jiang, J. Mater. Chem. A, 2015, 3, 4884-4891.

23 D. Wang, T. Zhou, J.-W. Zha, J. Zhao, C.-Y. Shi and Z.-M. Dang, J. Mater. Chem. A, 2013, 1, 6162-6168.

24 M. Li, X. Huang, C. Wu, H. Xu, P. Jiang and T. Tanaka, J. Mater. Chem., 2012, 22, 23477-23484.

25 C. Wu, X. Huang, L. Xie, X. Wu, J. Yu and P. Jiang, J. Mater. Chem., 2011, 21, 17729-17736.

26 X. Zeng, Y. Yao, Z. Gong, F. Wang, R. Sun, J. Xu and C.-P. Wong, Small, 2015, 11, 6205-6213.

27 J. Hu, Y. Huang, Y. Yao, G. Pan, J. Sun, X. Zeng, R. Sun, J.-B. Xu, B. Song and C.-P. Wong, ACS Appl. Mater. Interfaces, 2017, 9, 13544-13553.

28 R. Li, Z. Chen and J. Pei, Polymers, 2016, 8, 1-8.

29 X. Xu, B. Li, H. Lu, Z. Zhang and H. Wang, Appl. Surf. Sci., 2007, 254, 1456-1462.

30 H. Li, H. Tian, M. Cai, A. Gong, Z. Zhou, C. Wang and Z. Peng, 2017 International Symposium on Electrical Insulating Materials (ISEIM), 2017, pp. 149-152.

31 W. Cui, F. Du, J. Zhao, W. Zhang, Y. Yang, X. Xie and Y.-W. Mai, Carbon, 2011, 49, 495-500.

32 W. Yang, S. Yu, R. Sun and R. Du, Ceram. Int., 2012, 38, 35533562.

33 Z. Dai, B. Zhang, F. Shi, M. Li, Z. Zhang and Y. Gu, Appl. Surf. Sci., 2011, 257, 8457-8461.

34 M. Donnay, S. Tzavalas and E. Logakis, Compos. Sci. Technol., 2015, 110, 152-158.

35 J. Gu, Q. Zhang, J. Dang and C. Xie, Polym. Adv. Technol., 2011, 23, 1025-1028.

36 S. Chen, Q. Wang and T. Wang, Mater. Chem. Phys., 2011, 130, 680-684.

37 B. L. Zhu, J. Wang, H. Zheng, J. Ma, J. Wu, Z. H. Gan and J. Liu, J. Alloys Compd., 2017, 701, 499-507.

38 Y. Zhang, Y. Shen, K. Shi, T. Wang and E. Harkin-Jones, Composites, Part A, 2018, 110, 62-69. 